\title{
Performance measures for managing urban traffic signal systems
}

\author{
D. M. Bullock \& C. M. Day \\ Purdue University, West Lafayette, IN, USA
}

\begin{abstract}
Most urban traffic control systems go through a rather rigorous design phase based upon a set of fixed design volumes that do not capture the stochastic variation in traffic due to weather, incidents, special events, and shifting demand patterns. Once these systems are built, their operation is relatively open loop, with public feedback (complaints) often the primary feedback for assessing operations and initiating changes.

This paper describes procedures and case studies that illustrate how fundamental traffic engineering concepts can be integrated with traffic signal system detection and controller status information to provide real time performance measures. These performance measures characterize the operation of a traffic signal system and identify operation improvement opportunities. Several example performance measure graphics are provided to illustrate how these tools can be used for making operations decisions, evaluating field deployments, and preparing reports for decision makers. The paper concludes by discussing how these performance measures can be extended to include other surface transportation modes, particularly pedestrians, railroads, and transit.
\end{abstract}

Keywords: traffic signal, performance measure, priority, arterial.

\section{Introduction}

For the purpose of this paper, we propose the following definition for Traffic Signal Operation:

"Safe, efficient management of traffic signal infrastructure. Competing objectives, spanning multiple modes, are prioritized subject to engineering guidelines, budgetary constraints and local stakeholder input." 
For the past forty years, agencies and academics have implemented and modelled traffic signal systems with varying levels of success. Although all of these systems provide some level of reporting, none of these systems report performance measures in a manner that facilitates quantitative assessment of the system in either a systematic or visually intuitive format. The main reason for this performance measure deficiency has been the immature sensing and data collection infrastructure.

In recent years, the profession has reached a point where it is technologically possible to collect detailed data on signal operations, but little has been published defining how that data can be transformed into informative performance measures necessary for engineers to easily identify operational improvement opportunities. This paper reviews recent developments on both data collection and performance measurement, and argues that we must begin designing our traffic signal systems to collect high resolution data so we can construct graphical performance measure dashboards that are useful to engineering staff as well as easily understood by decision makers and the public. These performance measures provide tools to prioritize and answer the following questions:

1. Which intersections have the most capacity deficiencies?

2. At intersections with deficiencies, is there sufficient unused capacity at certain time periods of the day to mitigate deficiencies by reallocating green times?

3. At what periods of the day do those opportunities for mitigation occur?

4. During those time periods, which phases should be allocated additional capacity and which phases could perform acceptably with a reduced capacity allocation?

While traffic signal retiming will never replace the need for geometric or other network improvements, maintaining the most appropriate signal timing plans will improve usage of the existing infrastructure capacity, allowing agencies to do provide better service with fewer resources. Performance measures structured around the above four questions provide a framework for prioritizing traffic signal retiming efforts.

\section{Background}

Performance measures for an urban traffic signal system can be viewed as a multi-scale problem. At the macro-scale, one is interested in measuring system attributes such as passenger travel time. Once a portion of the system falls below a prescribed threshold, a more detailed set of performance measures is needed to locate the cause of system degradation, and perhaps identify opportunities for improving the system operation.

System travel time can be measured with techniques ranging from GPS [1] to probe tracking techniques that use a variety of emerging technologies [2] and the reporting techniques are relatively well defined. However, once an operational deficiency is identified in part of the system, detailed performance measures based upon high resolution traffic signal data must be developed to determine the cause. High resolution traffic signal data is defined as time-stamped log of all detector state changes (loop, pedestrian buttons, tram detection) and signal 
indication state changes (vehicles, pedestrian, and sometimes transit, tram, or rail indications). The time stamps are usually to the nearest 0.1 second [3] and can be used to calculate a variety of performance measures [4-8] based upon fundamental traffic engineering concepts [9]. By selecting the appropriate performance measure, one can identify traffic signal cycles [8], traffic signal offsets [4], or traffic signal green time allocation (splits) [5] requiring adjustment. The focus of this paper is on identifying opportunities to more effectively reallocate capacity by adjusting green times (splits).

\section{Real-time estimation of capacity utilization}

\subsection{Degree of phase saturation}

The capacity utilization of phase $\mathrm{i}$, can be estimated by the split utilization ratio $\left(\mathrm{X}_{\mathrm{i}}\right)$,

$$
X_{i}=\frac{3600}{s_{i}} \frac{N_{i}}{g_{i}}
$$
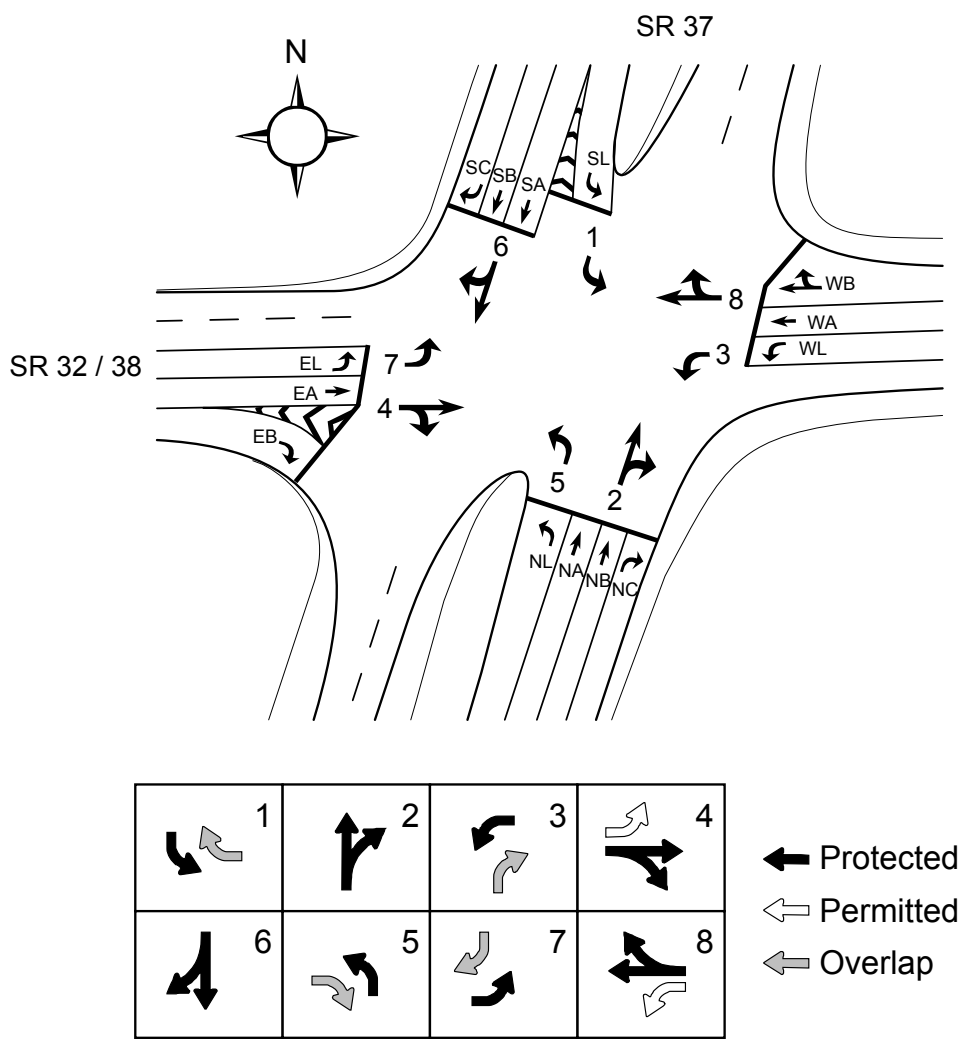

Figure 1: Phase labelling conventions. 
where:

$N_{i}=$ the vehicle count for phase $i$,

$s_{i}=$ the saturation flow rate for phase $i(\mathrm{veh} / \mathrm{h})$, and

$g_{i}=$ the effective green time for phase $i(\mathrm{~s})$.

Using the movement labelling convention (called phases) shown in Figure 1.

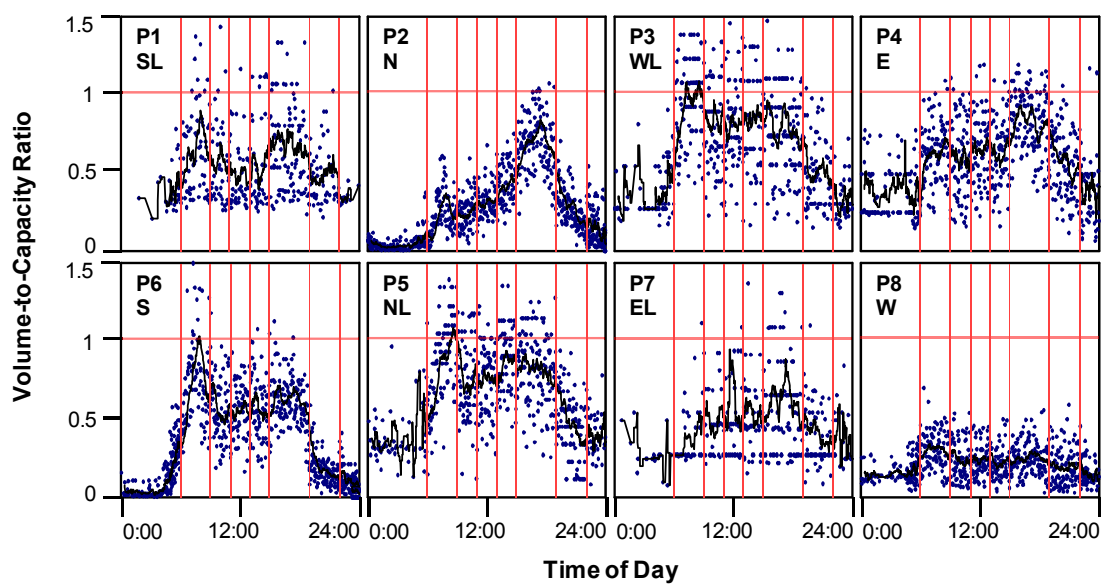

Figure 2: $\quad$ V/C Ratio for eight movements. The black lines show 20-point moving averages.

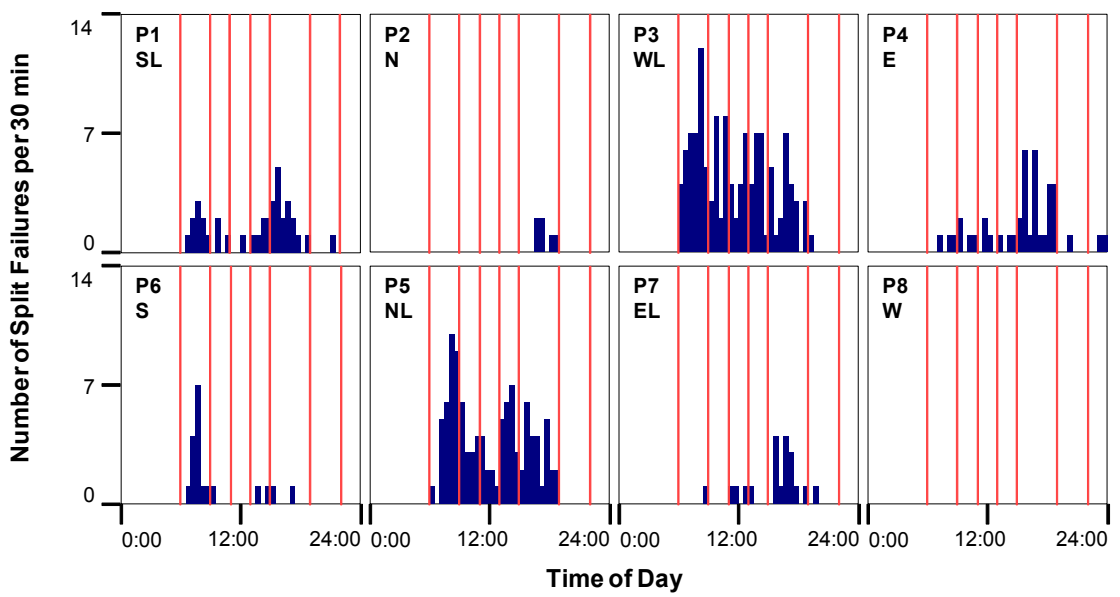

Figure 3: Number of estimated split failures per half hour bin for eight phases.

Figure 2 shows a 24-hour plot of $\mathrm{X}_{\mathrm{i}}$ for 8 movements (Left turns are the odd numbers and through movements are the even numbered phases). Assuming an arbitrary threshold of 1.0 for classifying phases as over capacity, a histogram of "split failures" can be prepared for each movement and is shown in Figure 3. 


\subsection{Degree of intersection saturation}

The degree of intersection saturation $\left(\mathrm{X}_{\mathrm{C}}\right)$, also called the critical volume-tocapacity ratio, is a measure described in the $\mathrm{HCM}$. The equation for $X_{C}$ is:

$$
X_{C}=\sum_{i}\left(\frac{V}{s}\right)_{c i}\left(\frac{C}{C-L}\right)
$$

where:

$C=$ cycle length (s),

$L=$ lost time (s), and

$\sum(\mathrm{v} / \mathrm{s})_{\mathrm{ci}}=$ the summation over critical phases $c i$ of the ratio of volume

(V) to saturation flow rate (s).

Figure 4 plots the overall intersection saturation over 24 hours for all phases. This plot tells the system operators when there is unused capacity within the existing cycle and there are opportunities to rebalance green allocations (say when $\mathrm{X}_{\mathrm{c}}<0.85$ ). It also tells the operator when there is little to no such opportunity. For example, between 16:00 and 18:00 there are several cycles that clearly have no reserve capacity $\left(\mathrm{X}_{\mathrm{c}}>1.0\right)$.

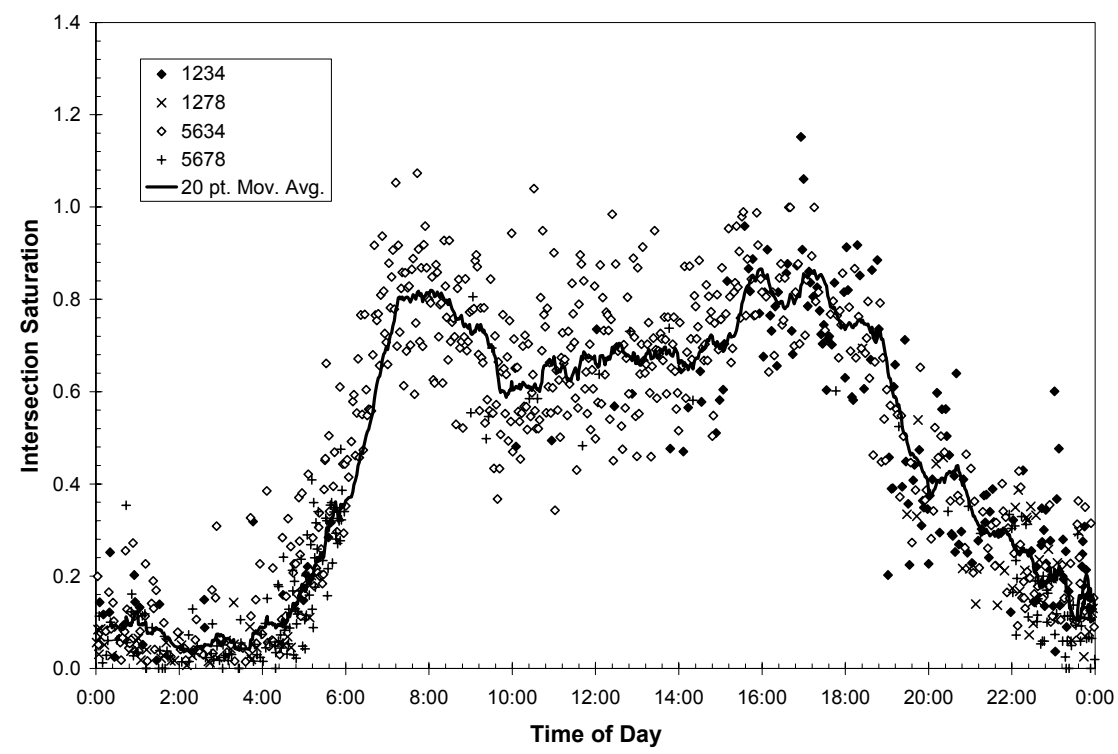

Figure 4: $\quad$ Plot of $X_{C}$ over 24 hours.

\subsection{Joining split failure indicators with intersection saturation}

Figure 5 plots the critical intersection saturation $\left(\mathrm{X}_{\mathrm{c}}\right)$ for cycles where an individual $X_{i}>1.0$. The symbol of the plotted $X_{c}$ value corresponds to the phase where $X_{i}>1.0$. From this, one can establish an upper bound on intersection 
saturation (say 0.85) for when an effort should be made to adjust the green allocation. From inspection of Figure 5, it is clear that during off peak periods there is opportunity to improve the performance of phase 3 . This is also substantiated by Figures 2 and 3 .

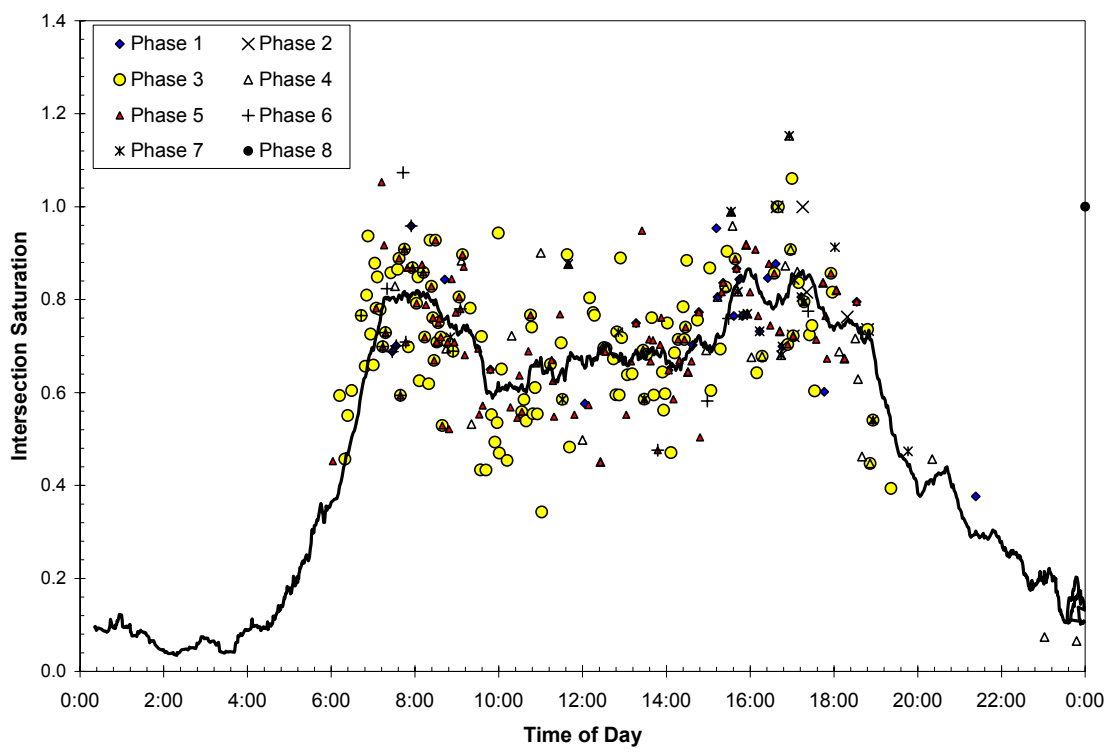

Figure 5: $\quad \mathrm{X}_{\mathrm{C}}$ graph with split failures.

\subsection{Prioritizing split failures at multiple intersections}

The distribution of split utilization ratios can be visualized by assembling $X_{i}$ for every phase of every cycle at an intersection and plotting it in descending order. For example, Trace "G" in Figure 6 shows that there approximately 40 occurrences when the split utilization ratio for Intersection $G$ exceeded 1.0. Alternatively, if one considers 0.90 to be critical, that threshold was exceeded approximately 80 times for Intersection $\mathrm{G}$. In contrast, Intersection $\mathrm{J}$ had no phases where $X_{i}$ exceeded 0.65 for any phase during any cycle during the day. Intersections $\mathrm{H}$ and I performed somewhere between $\mathrm{G}$ and $\mathrm{J}$.

\section{Prioritizing capacity reallocation}

Returning to our four questions:

1. What intersections have the most capacity deficiencies?

- Figure 6 provides a mechanism for identifying intersection $G$ as having the largest number of phases with capacity deficiencies. Figure 6 also illustrates there are no apparent capacity deficiencies for intersection J. 
2. At intersections with deficiencies, is there sufficient unused capacity at certain time periods of the day to mitigate deficiencies by reallocating green times?

- When one selects an intersection for analysis (Figure 5) it is clear that the intersection is operating below saturation outside the peak periods (07:00-09:00 and 16:00-18:00) with significant opportunities to make changes in green time reallocations.

3. At what periods of the day do those opportunities for mitigation occur?

- Figure 3 provides further detail, by time of day, in regards to what phases have the largest number of split failures that could benefit from green time reallocation.

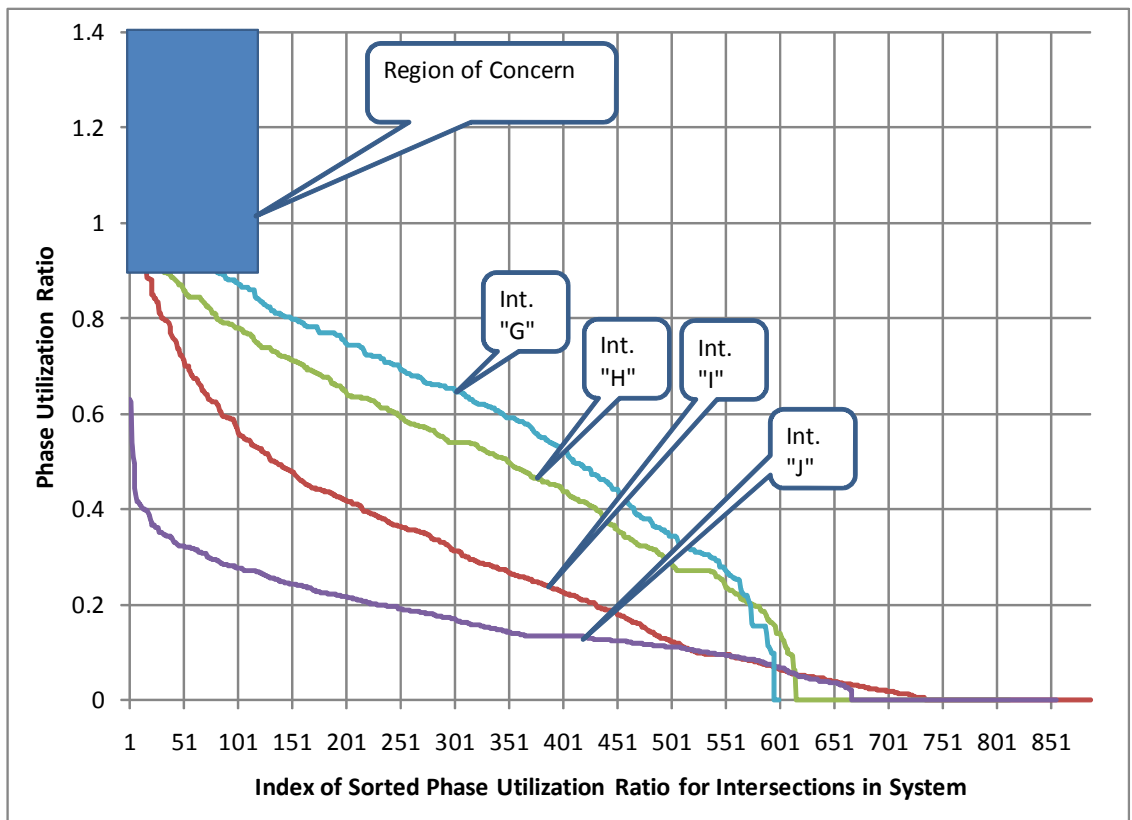

Figure 6: Rank ordering (descending) of phase utilization ratios, by intersection.

4. During those time periods, which phases should be allocated additional capacity and which phases could perform acceptably with a reduced capacity allocation?

- Figure 2 provides the cycle by cycle quantitative values for phase utilization ratios so that the system operator can make informed decisions with regard to what phases require additional capacity (green time) and what phases might relinquish some capacity (green time). For example, one could easily argue that phase 1 could take some green time from 
phase 2 during the morning and early afternoon periods to improve the performance of the movement. Similarly, phase 7 would benefit by receiving some additional green time from phase 8 and it is unlikely it would negatively impact phase 8 .

\section{Conclusions and recommendations}

This paper has developed, in a bottom up manner, a series of graphical performance measures that build from fundamental traffic engineering concepts (split utilization ratio) and are subsequently aggregated to allow longitudinal comparisons among multiple intersections in a system. The creation of theoretically sound intuitive visualization tools for documenting desirable/undesirable operating regimes provides a mechanism for traffic signal system operation personnel to use in a top-down fashion to:

1. Identify and prioritize intersections with capacity deficiencies, for subsequent evaluation.

2. Identify periods of the day where there is reserve capacity (that can be reallocated) at prioritized intersections.

3. Determine periods of the day where operational improvements can be made, and

4. Recommend candidate phases for capacity reallocation.

Lastly, after changes are made at selected intersections, performance measures would be relatively easy to re-tabulate to confirm that the modifications did in fact have the desired impact.

In conclusion, this paper focused on the vehicular traffic mode, but there are substantial opportunities for graphically intuitive, but theoretically sound, performance measures for pedestrian and rail interactions at signalized intersections $[6,7]$.

\section{References}

[1] Quiroga, C. and D. Bullock, "Travel Time Studies with Global Positioning and Geographic Information Systems: An Integrated Methodology," Transportation Research Part C, Pergamon Pres, Vol. 6C, No. 1/2, pp. 101127, 1998.

[2] Wasson, J.S., J.R. Sturdevant, D.M. Bullock, "Real-Time Travel Time Estimates Using MAC Address Matching," Institute of Transportation Engineers Journal, ITE, Vol. 78, No. 6, pp. 20-23, June 2008.

[3] Smaglik E.J., A. Sharma, D.M. Bullock, J.R. Sturdevant, and G. Duncan, "Event-Based Data Collection for Generating Actuated Controller Performance Measures," Transportation Research Record, \#2035, TRB, National Research Council, Washington, DC, pp.97-106, 2007.

[4] Smaglik, E., D.M. Bullock, and A. Sharma, "A Pilot Study on Real-Time Calculation of Arrival Type for Assessment of Arterial Performance," ASCE Journal of Transportation Engineering, Vol. 133, No. 7, pp. 415-22, July 2007. 
[5] Bullock, D.M., C.M. Day; J.R. Sturdevant, "Signalized Intersection Performance Measures for Operations Decision Making," Institute of Transportation Engineers Journal, ITE, Vol. 78, No. 8, pp. 20-23, August 2008 .

[6] Hubbard, S.M.L., D.M. Bullock, and C.M. Day, "Integration of Real-Time Pedestrian Performance Measures into Existing Traffic Signal System Infrastructure," Transportation Research Record, \#2080, TRB, National Research Council, Washington, DC, pp.37-47, 2008.

[7] Brennan, T.M., C.M. Day, D.M. Bullock, and J. R. Sturdevant," Performance Measures for Railroad Pre-empted Intersections," Transportation Research Board Paper ID: 09-0010, 2009.

[8] Day, C.M., D.M. Bullock, and J. R. Sturdevant," Cycle Length Performance Measures: Revisiting and Extending Fundamentals," Transportation Research Board Paper ID: 09-0061, in press, 2009.

[9] "Highway Capacity Manual 2000," Transportation Research Board, National Research Council Washington, D.C., 2000. 